Pesq. Vet. Bras. 37(4):385-388, abril 2017 DOI: $10.1590 / \mathrm{S} 0100-736 \mathrm{X} 2017000400013$

\title{
Eficácia da ivermectina comprimido no tratamento da sarna sarcóptica em cães naturalmente infestados 1
}

\author{
Gisele Maria de Andrade ${ }^{2 *}$, Moacir Marchiori Filho ${ }^{2}$, Marcelo Brunini ${ }^{3}$, \\ Armando Leonelo Neto ${ }^{2}$, Raquel A. Ré ${ }^{2}$, Alice T.S. de Matos ${ }^{2}$, Carlos R. \\ da Silva ${ }^{4}$ e Francisco de S.R. Carvalho ${ }^{4}$
}

\begin{abstract}
Andrade G.M., Marchiori Filho M., Brunini M., Leonelo Neto A., Ré R.A., Matos A.T.S., Silva C.R. \& Carvalho F.S.R. 2017. [Efficacy of the ivermectin tablet for treatment of sarcoptic mange in naturally infested dogs.] Eficácia da ivermectina comprimido no tratamento da sarna sarcóptica em cães naturalmente infestados. Pesquisa Veterinária Brasileira 37(4):385-388). Departamento de Pesquisa e Desenvolvimento, UCBVET Saúde Animal, Praça Dr. Joaquim Batista 150, Centro, Jaboticabal, SP 14870-090, Brazil. E-mail: gisele@ucbvet.com.br

The aim of this study was to evaluate the efficacy of the ivermectin tablet administered orally for treatment of Sarcoptes scabiei in naturally infested dogs. Fourteen 1 to 5 year-old Mongrel dogs presenting positive skin scrapings for $S$. scabiei mites, distributed into two groups with equal proportions of both sexes, containing seven animals per group were used in this study. All dogs were treated every 7 days, totaling four treatments in each dog (days $0,+7,+14$ and +21 ). Group I was administered the ivermectin ${ }^{5}$ tablet at a dose of $0.2 \mathrm{mg} / \mathrm{kg}$ and positive control group II as given an ivermectin commercial product at the same dose of group I. Skin scrapings, clinical and laboratorial parameters analysis were performed during the experimental period. Clinically there were no significant differences between the two groups evaluated prior and after treatments. Negative skin scrapings were observed in both groups from day +14 , remaining negative until the end of the experimental period. The initial skin lesions observed in clinical evaluation regressed from day +14 , and clinical improvement was evident in both groups. The ivermectin tablet (Ivermectan Pet, UCBVET Saúde Animal) administered orally was effective to treat S. scabiei infection in naturally infested dogs.
\end{abstract}

INDEX TERMS: Ivermectin, dogs, Sarcoptes scabiei, sarcoptic mange.

RESUMO.- 0 objetivo deste trabalho foi avaliar a eficácia da ivermectina comprimido administrada por via oral no tratamento de Sarcoptes scabiei em cães naturalmente infestados. Foram selecionados 14 cães com raspados cutâneos positivos para o ácaro S. scabiei,

\footnotetext{
${ }^{1}$ Recebido em 5 de abril de 2016.

Aceito para publicação em 13 de julho de 2016.

${ }^{2}$ Departamento de Pesquisa e Desenvolvimento (Farmacotécnico, Físico-Químico e Técnico), UCBVET Saúde Animal, Praça Dr. Joaquim Batista 150, Centro, Jaboticabal, SP 14870-090, Brasil. *Autora para correspondência: gisele@ucbvet.com.br

${ }^{3}$ Presidência, UCBVET Saúde Animal. Praça Dr. Joaquim Batista 150, Centro, Jaboticabal, SP 14870-090, Brasil.

${ }^{4}$ Gaia Pesquisa e Desenvolvimento em Saúde Animal, Av. Dr. Jaime Ribeiro da Luz 971, S.6, Bairro Santa Mônica, Uberlândia, MG 38408188, Brasil.
}

idade de 1-5 anos, sem raça definida, distribuídos na mesma proporção de ambos os sexos, em dois grupos experimentais, compondo 7 animais por grupo. Todos os animais foram tratados a cada 7 dias, totalizando quatro tratamentos em cada cão (Dias 0,7,14 e 21). No grupo I foi administrada a ivermectina ${ }^{5}$ comprimido na dosagem de $0,2 \mathrm{mg} / \mathrm{kg}$ e no grupo controle positivo (Grupo II) foi administrado um produto comercial a base de ivermectina comprimido na mesma dosagem do grupo I. Raspados cutâneos, avaliações clinicas e laboratoriais complementares dos cães foram realizadas durante o período de estudo. Clinicamente, não houve diferença significativa entre as avaliações antes e após o tratamento entre os dois grupos. Raspados negativos foram observados em ambos os grupos a partir do dia D+14, mantendo-se negativos até o final do período experi- 
mental. As lesões dermatológicas iniciais observadas no acompanhamento clínico regrediram e a partir do dia D+14 a melhora clínica era evidente em ambos os grupos. A ivermectina (Ivermectan Pet, UCBVET Saúde Animal) administrada por via oral foi eficaz no tratamento de S. scabiei em cães naturalmente infestados.

TERMOS DE INDEXAÇÃO: Ivermectina oral, cães, Sarcoptes scabiei, sarna sarcóptica.

\section{INTRODUÇÃO}

A estreita relação entre o homem e animais necessita de cuidados constantes, levando-se em consideração que estes frequentam sua moradia ou habitam locais próximos a ela, aumentando os riscos da ocorrência de zoonoses (Dryden 1993). Além disso, os endoparasitos e os ectoparasitos podem gerar prejuízos e causar danos severos para a saúde do animal (Taylor 2001). A sarna sarcóptica, também conhecida como escabiose, é uma dermatose parasitária causada pelo ácaro Sarcoptes scabiei. Doença parasitária altamente contagiosa entre os animais domésticos como cães, gatos, equinos, ovinos, caprinos, bovinos, coelhos e roedores, com potencial zoonótico podendo infestar o ser humano (Heukelbach \& Feldmeier 2006, Pinchbeck \& Hillier 2008). Em cães, a sarna sarcóptica é causada pelo $S$. scabiei variedade canis. O contágio se dá por contato direto com animais infectados principalmente, podendo haver transmissão por fômites e pele infestada de animais por meio de contato com material de higienização ou em canis (Pinchbeck \& Hillier 2008). A doença manifesta-se com prurido intenso na pele, formando crostas hemorrágicas, perda de pelos e provocando o aparecimento de feridas (Piccinin et al. 2008). Os ácaros afetam preferencialmente as áreas de pelagem mais esparsa como as orelhas, focinho, cabeça e pescoço. As escavações provocam reações inflamatórias, prurido, espessamento da pele, perda de pelos (alopecia), aumento da descamação, e o prurido intenso que pode levar a escoriações, hemorragias, e infecções secundárias (Wall \& Shearer 2001).

Vários produtos antiparasitários vêm sendo usados para o tratamento de sarna sarcóptica, com formulações prontas para uso ou não, que variam desde múltiplas aplicações tópicas a dose única injetável (Argüello et al. 2001). A ivermectina, uma avermectina, produzida através da fermentação do actinomiceto do solo Strepotomyces avermitilis (Carrasco et al. 1997, Scott et al. 2002) foi disponível comercialmente em vários países a partir de 1981 e introduzida na medicina veterinária para o controle de parasitas em bovinos, equinos, ovelhas e suínos (Fadok et al. 1984, Martineau et al. 1984, Foil \& Foil 1986, Barragry et al. 1987). Tem amplo espectro de ação interferindo no sistema nervoso de nematoides e artrópodes que parasitam os animais. Embora altamente ofensivo aos insetos, os mamíferos geralmente não são afetados pelas doses terapêuticas das formulações das avermectinas (Clark et al. 1995). Seu uso em cães e gatos é aprovado para prevenção da dirofilariose (Mueller 2004), mas é muito útil na der- matologia de pequenos animais no tratamento de várias infestações e, no manejo de outras alterações cutâneas relacionadas à ecto e endoparasitos (Paradis 1998). A absorção da ivermectina sistêmica obtém melhor eficácia e maior meia vida quando utilizada por via parenteral ou transdérmica (Taylor 2001), sendo a via oral indolor e de maior praticidade, principalmente quando a administração do produto é feita pelo proprietário (Coelho et al. 2014). O objetivo do presente estudo foi avaliar a eficácia da ivermectina oral no tratamento de cães naturalmente infestados pela sarna sarcóptica.

\section{MATERIAL E MÉTODOS}

Este estudo foi aprovado pelo Comitê de Ética de Uso Animal (CEUA) da empresa Gaia com número de protocolo 021/032012. Quatorze cães (7 machos e 7 fêmeas), idade entre um e cinco anos, sem raça definida (SRD), peso de 5,1 a 18,6 kg, que apresentaram diagnóstico clinico e laboratorial positivos para Sarcoptes scabiei oriundos da Associação de Proteção Animal (APA)/Uberlândia -MG e da casuística de animais atendidos no setor de Atendimento Clínico do Hospital Veterinário da Universidade Federal de Uberlândia (UFU) foram selecionados para este estudo. 0 diagnóstico inicial foi realizado através de raspados de pele em áreas com lesão de $1 \mathrm{~cm}^{2}$ nos dias D-7, D-1 e D-2, além da realização de exames complementares: hemograma e bioquímico. Os animais foram divididos aleatoriamente em dois grupos de sete animais cada.

O Grupo I foi tratado, via oral, com ivermectina comprimido $^{5}$ (Ivermectan Pet, UCBVET Saúde Animal) na dose de 0,2mg/ $\mathrm{kg}$. Os animais do grupo II (controle positivo) foram tratados, via oral, com ivermectina comprimido (produto comercial) na dose $0,2 \mathrm{mg} / \mathrm{kg}$. Todos os cães foram tratados a cada 7 dias, durante 21 dias, completando assim um total de quatro tratamentos para cada animal (dias 0, 7,14 e 21). Todos os animais foram pesados para calcular a dose a ser administrada, e a cada nova administração, o peso foi mensurado novamente e a dose ajustada.

Os animais provenientes da APA foram mantidos em canis com solário e os animais atendidos no HV da UFU foram mantidos na respectiva residência. Todos os cães foram alimentados com ração comercial, ou comida caseira, de acordo com a rotina adotada por seus proprietários, e água a vontade. Todos os cães tinham histórico prévio de não ter recebido nenhum tratamento antiparasitário inclusive sarnicida.

Avaliações clínicas (exames clínicos: temperatura retal, frequência cardíaca, respiratória e avaliação do pelame) e avaliações hematológicas, bioquímicas e raspado cutâneos para acompanhamento clinico durante o período de estudo foram realizados de acordo com o as informações contidas no Quadro 1.

A avaliação quanto à presença de $S$. scabiei nos animais nos períodos determinados foi realizada através de raspados cutâneos nas áreas de alopecia utilizando-se laminas de bisturi, e duas áreas de no mínimo $1 \mathrm{~cm}^{2}$, de acordo com a técnica descrita por Silva et al. (2008). O material colhido foi depositado em lamina de vidro com uma gota de óleo mineral, coberto por lamínula, e observada em microscópio óptico em objetiva de 40 ou 100x, para determinação entre ácaros vivos e mortos (Silva et al. 2008). A eficácia foi avaliada pela remissão dos sintomas, desaparecimento das lesões e dos ácaros nos esfregaços cutâneos.

Os resultados obtidos na avaliação dos parâmetros clínicos e hematológicos foram comparados estatisticamente utilizando-se analise de variância e posteriormente teste de Tukey nível de 5\% 
através do programa INSTANT (1988). Os resultados dos exames de raspado cutâneo foram analisados por comparação entre o grupo I e II.

\section{RESULTADOS E DISCUSSÃO}

Os resultados das análises estatísticas revelaram que não houve diferença estatística significativa $(\mathrm{p}<0,05)$ nos valores médios dos parâmetros clínicos e hematológicos entre os grupos I e II. Na avaliação do pelame de todos os cães no dia D-7, foi observada área de alopecia, presença de crostas

Quadro 1. Avaliações realizadas nos cães pertencentes aos grupos controle e tratado durante o período experimental

\begin{tabular}{lc}
\hline Avaliações & Momento experimental (dias) \\
\hline Parâmetros clínicos & D-7, D0, D+3, D+7, D+14, D+21, D+28 e D+35 \\
$\begin{array}{ll}\text { Parâmetros hematológicos } & \text { D-7, D+7, D+28 } \\
\text { Raspado cutâneo } & \text { D-7, D-2, D-1,D+3, D+7,D+14, D+21, D+28 e D+35 }\end{array}$
\end{tabular}

Quadro 2. Presença de Sarcoptes scabiei em raspado cutâneo dos cães pertencentes aos grupos controle e tratado com ivermectina oral, antes e depois do tratamento

\begin{tabular}{lllllllllll}
\hline Grupo/Cão & \multicolumn{7}{c}{ Presença de Sarcoptes scabiei/Dia experimental } \\
\cline { 2 - 8 } & D-7 & D-2 & D-1 & D+3 & D+7 & D+14 & D+21 & D+28 & D+35 \\
\hline Controle & & & & & & & & & \\
70253 & + & + & + & + & + & - & - & - & - \\
71009 & + & + & + & + & + & - & - & - & - \\
71788 & + & + & + & + & + & - & - & - & - \\
70017 & + & + & + & + & + & - & - & - & - \\
70300 & + & + & + & + & + & - & - & - & - \\
70433 & + & + & + & + & + & - & - & - & - \\
71912 & + & + & + & + & + & - & - & - & - \\
Tratado & & & & & & & & & \\
71518 & + & + & + & + & + & - & - & - & - \\
1013 & + & + & + & + & + & - & - & - & - \\
71814 & + & + & + & + & + & - & - & - & - \\
71133 & + & + & + & + & + & - & - & - & - \\
70715 & + & + & + & + & + & - & - & - & - \\
70505 & + & + & + & + & + & - & - & - & - \\
70080 & + & + & + & + & + & - & - & - & - \\
Eficácia (\%) & & & & & & $100 \%$ & $100 \%$ & $100 \%$ & $100 \%$
\end{tabular}

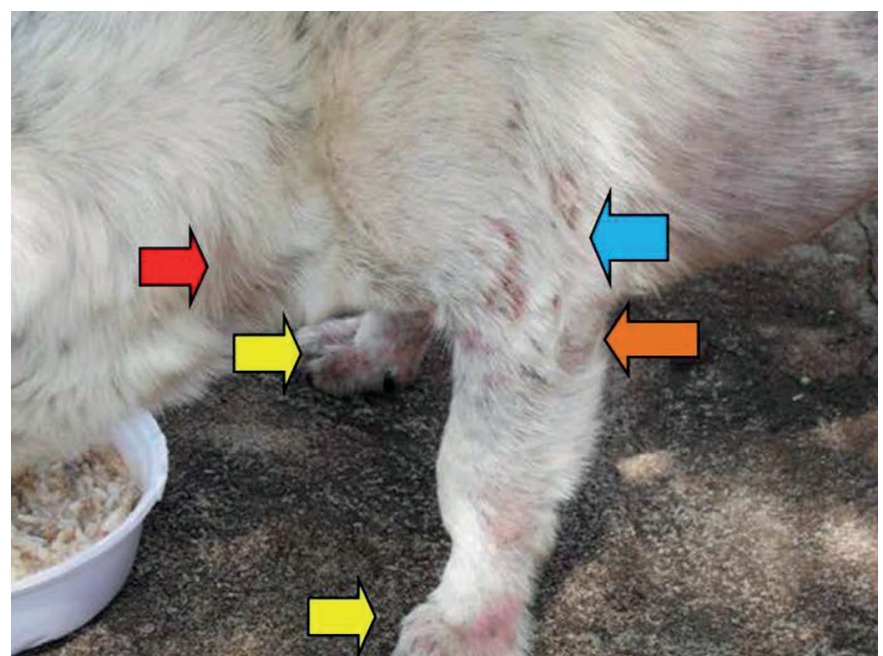

Fig.1. Cão 70080, grupo I tratado, no momento experimental D-7. Observar região com eritema e alopecia no pescoço (seta vermelha), dedos (setas amarelas), próximo à região do cotovelo (seta azul), cotovelos (seta laranja).

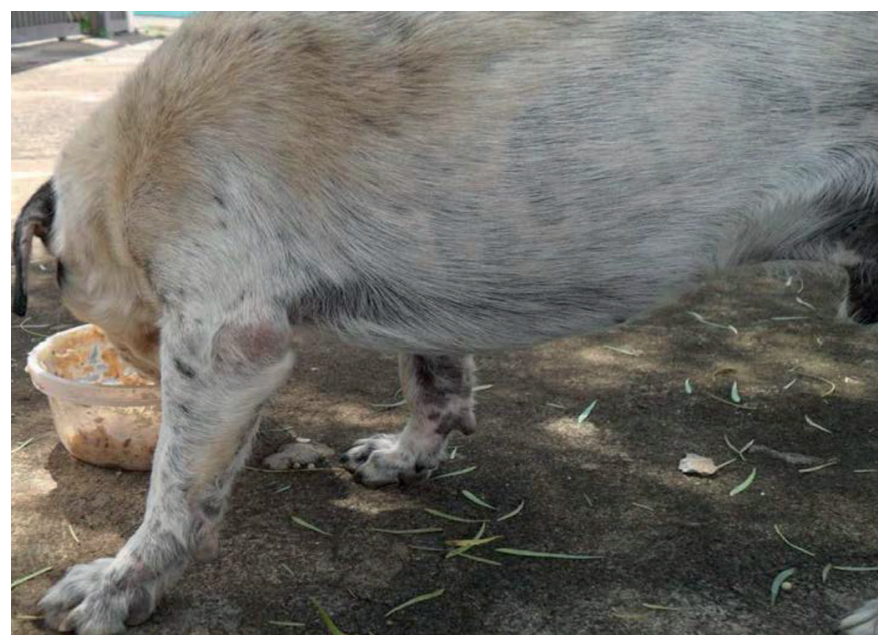

Fig.2. Cão 70080, grupo I tratado, no momento experimental $\mathrm{D}+28$. Observar as regiões anteriormente acometidas pelo ácaro como cotovelos e dedos com crescimento de pelo e ausência de eritema.

e escamas, espessamento da pele, escoriações resultantes do ato de coçar, hiperemia e prurido. As lesões se localizaram em grande parte nas regiões das bordas das orelhas, cotovelos, calcanhares e dedos. No dia D-7, fichas dermatológicas determinando o local das lesões a as características das mesmas foram preenchidas para cada animal (Fig.1). A partir do D+14, as lesões dermatológicas apresentaram melhoras evidentes com diminuição de eritema, prurido, e crescimento de pelos nas áreas alopécicas nos animais do grupo I e II. No dia +28 , foi observado remissão dos sinais clínicos em 100\% dos animais (Fig.2).

Os resultados dos animais do grupo controle positivo e grupo tratado com ivermectina oral no tratamento de Sarcoptres. scabiei encontram-se no Quadro 2. Nos dias $-7,-2,-1,+3$ e +7 todos os animais do estudo apresentaram resultados positivos para $S$. scabiei. Resultados negativos para $S$. scabiei foram observados nos dias $+14,+21,+28$ e +35 . Resultados semelhantes foram encontrados em um estudo terapêutico com cães realizado na cidade de Ribeirão Claro em 2010, onde se observou 100\% de cura dos animais infectados após algumas semanas de tratamento com Ivermectina, via oral, uma vez por semana (Ruiz \& Francisco 2010). Em outro estudo, Scheidt et al. (1984) avaliaram a eficácia da ivermectina oral na dose $0,2 \mathrm{mg} / \mathrm{kg}$ em 298 cães. Foram realizados dois tratamentos com intervalo de 14 dias, e 28 dias pós-tratamento e foi observada a cura de todos os animais pelos resultados negativos para $S$. scabiei nos raspados cutâneos.

Paradis (1998) mostrou que a partir de estudos realizados em cães, que o regime de tratamento mais adequado para animais infestados com $S$. scabiei seria pela

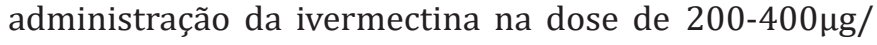
$\mathrm{kg}$, via oral a cada 7 ou via SC a cada 14 dias, e por 4 a 6 semanas de tratamento.

Neste experimento não foi observado nenhum efeito adverso relacionado ao tratamento durante o período de avaliação deste estudo. 


\section{CONCLUSÃO}

A ivermectina (Ivermectan Pet, UCBVET Saúde Animal) na dose de $0,2 \mathrm{mg} / \mathrm{kg}$, administrada por via oral em quatro doses com intervalo de 7 dias, foi eficaz no controle de Sarcoptes scabiei em cães naturalmente infestados.

\section{REFERÊNCIAS}

Argüello H.M.R., Iez-Baños N.D., Martinez-Gonzáles B. \& Rojovásquez F.A. 2001. Efficacy of moxidectin 1\% against natural infection of Sarcoptes scabiei in sheep. Vet. Parasitol. 102:143-150.

Barragry T.B. 1987. A review of the pharmacology and clinical use of ivermectin. Can. Vet. J. 28:512-517.

Carrasco M.S.D., Espuny A., Escudero E. \& Cárceles Y.C.M. 1997. Farmacologia de los endectocidas: aplicaciones terapêuticas. Anales Vet. Murcia 13(14):3-22.

Clark J.M., Scott J.G., Campos F. \& Bloomquist J.R. 1995. Resistance to avermectins: extent, mechanisms, and management implications. Ann. Rev. Entomol. 40:1-30.

Coelho C.N., Nunes T.A.P., Batista L.C.S.O., Silva D.D., Santos R.R., Correia T.R., Scott F.B. \& Fernandes J.I. 2014. Eficácia da ivermectina oral no controle de Psoroptes ovis e Leporacarus gibbus em coelhos naturalmente infestados. Pesq. Vet. Bras. 34(9):832-836.

Dryden M.W. 1993. Biology of fleas of dogs and cats. Compendium Cont. Educ. Pract. Vet. 15:569-579.

Fadok V.A. 1984. Parasitic skin diseases of large animals. Vet. Clin. North Am., Large Anim. Pract. 6:3-26.

Foil L. \& Foil C. 1986. Parasitic skin diseases. Vet. Clin. North Am., Equine Pract. 2:403-437.
Heukelbach J. \& Feldmeier H. 2006. Scabies. Lancet 367:1767-1774.

Martineau G.P., Vaillancourt J. \& Frechette J.L. 1984. Control of Sarcoptes scabiei infestation with ivermectin in a large intensive breeding piggery. Can. Vet. J. 25:235-238.

Mueller R.S. 2004. Treatment protocols for demodicosis: an evidence-based review. Vet. Dermatol. 15(1):75-89.

Paradis M. 1998. Ivermectin in small animal dermatology. II. Extralabel applications. Compen. Contin. Educ. Pract. Vet. 20(4):459-469.

Piccinin A., Ferrari M.L.O.P., Prado M.O. \& Spigolon Z. 2008. Sarna sarcóptica em cães. Revta Cient. Eletrôn. Med. Vet., Garça/SP, 7(10).

Pinchbeck L.R. \& Hillier A. 2008 Escabiose, Sarna notoédrica e queiletielose, p.473-478. In: Birchard S.J. (Ed.), Manual Saunders: clínica de pequenos animais. $3^{\underline{a}}$ ed. Roca, São Paulo.

Ruiz S.V. \& Francisco O. 2010. Estudo terapêutico da sarna sarcóptica em cães na cidade de Ribeirão Claro-PR. Anais IX Congresso de Iniciação Científica das Faculdades Integradas de Ourinhos, SP.

Scott F.B., Martins I.V.F., Souza C.P. \& Correia T.R. 2002. Aspectos gerais do controle da pulga Ctenocephalides felis felis em cães. Hora Vet. 125(1):13-18.

Silva R.P.B., Belettini S.T., Stel R.F., Martins L.A. \& Pachaly J.R. 2008. Sarna demodécica canina e suas novas perspectivas de tratamento: revisão. Arq. Ciênc. Vet. Zool. Unipar, Umuarama, 11(2):139-151.

Scheidt V.J., Medleau L., Seward R.L. \& Schwartzman R.M.1984. An evaluation of ivermectin in the treatment of sarcoptic mange in dogs. Am. J. Vet. Res. 45(6):1201-1202.

Taylor M.A. 2001. Recent developments in ectoparasiticides. Vet. J. 161(3):253-268.

Wall R. \& Shearer D. 2001. Veterinary Ectoparasites: biology, pathology and control. 2nd ed. Blackwell Publishing Ltd, Oxford, UK. 